analysis of large-scale social interactions in a way that reveals something about how trust functions in them is a fascinating direction for research.

But for such research to flourish, it must engender that which it seeks to describe. And so it is encouraging that computational social scientists are trying to anticipate threats to trust that are implicit in their work. Any data on human subjects inevitably raise privacy issues (see page 644), and the real risks of abuse of such data are difficult to quantify. But although the risks posed by researchers seem far lower than those posed by governments, private companies and criminals, their soul-searching is justified. Abuse or sloppiness could do untold damage to the emerging field.

Rules are needed to ensure data can be safely and routinely shared among scientists, thus avoiding a Wild West where researchers compete for key data sets no matter what the terms. The complexities of anonymizing data correctly, and the lack of experience of local ethical committees in such matters, calls for an institutionalized approach to setting standards and protocols for using personal data, as rightly recommended recently by the US National Academy of Sciences. Solid and well thought out rules for research are essential for building trust.

But researchers are right to argue that better protection ultimately lies in protecting electronic privacy more broadly. At present, privacy legislation in most countries lags far behind what is actually happening online. Companies and governments are insufficiently liable for abuse of the data they collect. Deliberate abuse of phone, e-mail, financial, medical and other personal records held should be made a criminal offence.

"Trust is of crucial
importance to the
contract between
scientific expertise and
the broader society
that supports it."

Only by restoring a sense of control and ownership to the data subjects can better electronic trust be established. And only then will people come to feel more comfortable with the idea of research being done on their intimate details of their anonymized search results, e-mails, movements and telephone calls.

\section{How to be good?}

\section{Mentoring and training for ethical behaviour aren't all they're cracked up to be.}

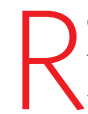
esearchers have always depended on their seniors to convey the peculiar knowledge of the lab. Techniques, values, scientific judgement and survival skills are imparted by good mentors at the bench and through challenging discourse at lab meetings or in the local pub. Young scientists enjoying such inductions are popularly viewed as the lucky ones, as opposed to those reared on the 'sink or swim' principle.

Some particularly grateful protégés offered nominations for Nature's annual competition to find outstanding mentors, this year held in South Africa, and we are happy to congratulate the winners (see page 752). But the glow of a job well done should not stop us from asking questions about mentoring in general. Just how effective is it? In particular, do young scientists behave better as a result?

Melissa Anderson and her colleagues at the University of Minnesota in Minneapolis decided to investigate the relative effects of mentoring and formal instruction in setting a young scientist's ethical framework. They analysed the 3,250 respondents from a 2002 survey of about 6,900 grantees of the US National Institutes of Health (NIH) who were asked about their formal ethics instruction and the informal mentoring they had received - and how these had affected their subsequent behaviour (M. S. Anderson et al. Acad. Med. 82, 853-860; 2007).

It turns out that, despite the NIH requiring formal training in the responsible conduct of research since 1990, it was still lacking ten years later. As many as a quarter of the NIH PhD fellows in 2000-01 had not taken ethics courses or been mentored in ethics workshops or discussion roundtables. One quarter of the survey respondents admitted that they did not feel well prepared to deal with ethical issues in their work.
More positively, about $90 \%$ of both early- and late-career respondents had discussed ethics with their mentors or colleagues and had been mentored in good research practices. Among younger scientists, biologists were among the least likely to have been mentored in ethics. It seems they received more mentoring in getting financial support for their work. Social and physical science postdocs were more likely to be mentored in how to survive in their fields and develop professional relationships.

So does the extent of mentorship and/or formal ethics training correlate with behaviour? The survey asked participants to report various types of problematic behaviours. Formal instruction exerted a disappointing influence on the early-career scientists: in fact, it significantly increased the odds of poor choices when collecting and analysing data, dealing with other researchers' confidential information or allowing funders inappropriate influence. Formal training was also correlated with a higher likelihood of not giving proper credit to others. How could this be? Perhaps such courses introduce scenarios that were unimaginable beforehand while suggesting that others have got away with such behaviours.

The results of mentoring early-career scientists were better but still mixed. Research mentoring (teaching good practice and presentation of one's results) and ethics mentoring decreased the likelihood of bad behaviour in almost all categories. But receiving mentoring advice on how to survive in the field and form professional relationships, or on how to support one's research, increased misbehaviour.

Collective discussions are perhaps a better way to reinforce good behaviour. A good forum for such discussions might be the lab's journal club - particularly if the mentor is skilled in inviting open discussion in a non-confrontational atmosphere. Mentors who regularly set aside time to discuss issues of concern may find that everyone benefits from the 'wisdom of the crowd'. Students can be asked to propose topics to kick off discussion: "I have a friend whose adviser asked her to write several anonymous reviews for him because he is too busy. Should she say yes? Ask for proper attribution?" Figuring it out together is the way to raise everyone's game. 